\title{
Article
}

\section{Deep Learning Exoplanets Detection by Combining Real and Synthetic Data}

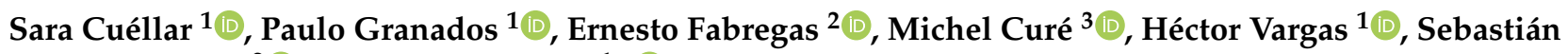 \\ Dormido-Canto $^{2}$ (D) and Gonzalo Farias ${ }^{1, *(D)}$ \\ 1 Escuela de Ingeniería Eléctrica, Pontificia Universidad Católica de Valparaíso, Av. Brasil 2147, 2362804 \\ Valparaíso, Chile; milahcr@hotmail.com \\ 2 Departamento de Informática y Automática, Universidad Nacional de Educación a Distancia, Juan del Rosal \\ 16, 28040 Madrid, Spain; efabregas@dia.uned.es \\ 3 Instituto de Física y Astronomía. Facultad de Ciencias. Gran Bretaña 1111. Playa Ancha. Valparaíso. Chile; \\ michel.cure@uv.cl \\ * Correspondence: gonzalo.farias@pucv.cl; Tel.:+56-32-2273673
}

\begin{abstract}
Scientists and astronomers have attached Scientists and astronomers have attached great importance to the task of discovering new exoplanets, even more so if they are in the habitable zone. To date, more than 4300 exoplanets have been confirmed by NASA, using various discovery techniques, including planetary transits, in addition to the use of various databases provided by space and ground-based telescopes. This article proposes the development of a deep learning system for detecting planetary transits in Kepler Telescope lightcurves. The approach is based on related work from the literature and enhanced to validation with real lightcurves. A CNN classification model is trained from a mixture of real and synthetic data, and validated only with real data and different from those used in the training stage. The best ratio of synthetic data is determined by the perform of an optimisation technique and a sensitivity analysis. The precision, accuracy and true positive rate of the best model obtained are determined and compared with other similar works. The results demonstrate that the use of synthetic data on the training stage can improve the transit detection performance on real light curves.
\end{abstract}

Keywords: Exoplanets Detection; Deep learning; Real and Simulated Data.

\section{Introduction}

All the planets in our solar system orbit the sun. Planets orbiting other stars are called exoplanets under NASA's Exoplanet Exploration Program [1].

Exoplanets are very difficult to see directly with telescopes. They are hidden by the brightness of the stars they orbit. The search for planets outside the solar system has been investigated for many years. The existence of a possible exoplanet orbiting the white dwarf Van Maanen 2 has been suspected since 1917 [2], but its existence could not be confirmed due to the limited technology of the time.

It was not until 1995 that Michel Mayor and Didier Queloz first confirmed an exoplanet called Dimidium or 51 Pegasi, with a 4-day orbit around the nearby star Helvetios [3]. They described it as a large ball of gas similar to Jupiter. For this finding they received the Nobel Prize in Physics 2019 [4].

Nowadays, scientists and astronomers have attached great importance to the task of discovering new exoplanets, even more so if they are in the habitable zone. Most of the exoplanets discovered so far are found in a relatively small region of our galaxy, the Milky Way. To date, NASA has confirmed 4301 exoplanets, using a variety of discovery techniques [5], including planetary transits, radial velocities, gravitational microlensing and direct imaging from databases provided by space and ground-based telescopes, e.g. 
NASA's Kepler space telescope [6] and the NASA's Transiting Exoplanet Survey Satellite (TESS) [7].

The Kepler space telescope has collected data on a large number of stars (in the order of 200,000) during the 4 years it was operating (2009-2013). The manual analysis of all these light curves is a time-consuming work. In this context, the use of artificial intelligence methods have emerged as tools for the analysis of this information.

In the literature, different approaches that use artificial intelligence techniques to detect exoplanets can be found. For example, in [8], the authors describe a method for detecting exoplanet transits by applying the k-nearest neighbors $(\mathrm{kNN})$ method to determine whether a given signal is sufficiently similar to known transit signals. In [9], they present for the first time the use of the Random Forest Classifiers (RFCs) algorithm for exoplanets classification. They achieve an overall error rate of $5.85 \%$ and an error rate in the classification of exoplanet candidates of $2.81 \%$. The work described in [10], shows a combination of RFCs and Convolutional Neural Networks (CNNs) to distinguish between the different types of signals. The authors say that the combination of both methods offers the best approach to identify exoplanets correctly in the test data approximately $90 \%$ of the time. While in [11], the authors present another $\mathrm{CNN}$ based approach that is capable of detecting Earth-like exoplanets in noisy time series data with a greater accuracy than a least-squares method. The most important disadvantage of this case is that they do not use real traffic data to train the model. This does not provide evidence for its performance against real data.

In [12], the method for classifying candidates using a Self-Organizing Maps (SOM) technique is developed on Kepler and $\mathrm{K} 2$ confirmed and candidate planets with a success of 87\%. More recently, in [13] an Ensemble-CNN model for exoplanets detection is presented with an accuracy of $99.62 \%$.

Other approaches such as [14], shows a 98\% cross-validated precision score using RFCs to classify objects of interest in Kepler's cumulative information object table. But, in this case, the authors use only data from the training stage for cross-validation of their models. This does not allow to properly analyse the performance of the model with new data.

Despite the good results obtained by these previously mentioned works, most of them show that in order to build and validate the models, in some cases light curves of unconfirmed planet candidates are used or even some of them are false positives.

The main contribution of this work is the development of a system for detecting planetary transits in Kepler Telescope light curves. This system includes the generation of synthetic data from estimated parametric models of the planet candidate. The exoplanet detection model is trained by deep learning from a mixture of real and synthetic data. This model consists on building an image from the folding of light curves. This image is used to determine planetary transits by means of a CNN (Xception referencing). On the other hand, the validation of the model is only performed with real data and different from those used in the training stage. This shows that the performance of the model is better than if only real data are used for training. In addition, a sensitivity analysis and an optimisation technique is performed to determine the best ratio of synthetic data. Finally, this approach allows finding planetary transits over a wider range of periods.

The rest of the paper is structured as follows. Section 2 presents some exoplanet detection approaches that can be found in the literature and describes briefly the approach which is the start point of this work. Section ?? details the proposed method. Section 3 shows the experimental results and a comparison with previous results. Finally, Section 4 summarizes the main conclusions and future work.

\section{Exoplanets detection approaches}

As mentioned above, the discovery of new exoplanets has taken a high degree of importance during the last few years. Since the amount of data provided by telescopes is enormous, it is much better if this is analyzed using Machine Learning techniques. A significant amount of research can be found in the literature that has focused on the use of 
Machine Learning techniques for exoplanet detection. This section presents a small review of these approaches.

Table 1 presents a summary of the articles covered in this brief review. The first column contains the reference to the article in the bibliography. The second column shows the name of the telescope/catalog from which the data were obtained. The third contains the name of the data set provided by the telescope/mission and used for the investigation. The fourth column shows the details about the feature extraction used. The fifth column shows the machine learning method used for detection. Sixth column shows the dataset used to train the model. The seventh contains the data used for testing and finally the eighth shows the results obtained.

In [15], published in 2015, the authors present the Autovetter, a machine learning based classifier. It is used to produce a catalog of Planet Candidates from the Q1-Q17 DR24 Threshold Crossing Events (TCEs) that are identified in the Kepler Science Operations Center pipeline. The Autovetter classify TCEs into three classes: 1.- Planet Candidate (PC), which contains signals that are consistent with transiting planets; 2.- Astrophysical False Positive (AFP), which contains signals of astrophysical origin that could mimic planetary transits; and 3.- Non-Transiting Phenomenon (NTP), which contains signals that are evidently of instrumental origin, or are noise artifacts. A set of 114 atributes calculated from Kepler pipeline are ultimately used to build a random forest classifier that maps the attributes of any TCE to a predicted class label of either PC, AFP, or NTP. The results show the following accuracy/error rate for each class: PC $(0.971 / 2.9 \%)$, AFP $(0.976 / 2.4 \%)$ and NTP $(0.968 / 3.2 \%)$. As can be seen, the results are very accurate, in fact, the Autovetter predictions are taken as ground truth for posterior studies.

In [16], the authors present a method for classifying potential signals from planets using deep learning, specifically convolutional neural networks (CNNs). Feature extraction is generated by folding each flattended light curve in the TCE period (with the event centered) and clustering to produce a 1D vector. The training and test sets (PC, AFP and NTP) were selected from the Autovetter Planet Candidate Catalog for Q1-Q17 DR24. The result is a CNN model named Astronet that is able to distinguish with good accuracy the subtle differences between genuine transiting exoplanets and false positives such as eclipsing binaries, instrumental artifacts, and stellar variability. They also compared models based on linear logistic regression (LLR) and a fully connected neural network. The results show a performance of classified real planets with $95 \%$ recall, $90 \%$ of accuracy and $96 \%$ of precision.

In [17] the authors also present an approach based on CNN named Exonet. They use a dataset from the same catalog as the previous one (Kepler Q1-Q17 DR24). For the classification process, they use phase-folded light curves and associated centroid curves (measured by the Kepler pipeline from the same TPF), for both global and local views. They also add stellar normalized parameters like: effective temperature, surface gravity, metallicity, radius, mass, and density to the training set. The results overperformed the Astronet with an accuracy of $97.5 \%$ and $95.5 \%$ of precision.

In [18], the first deep neural network trained and tested on real TESS data is presented. The model is modified based on Astronet and designed to automatically performing triage and vetting on TESS candidates. In triage mode, it can distinguish transit-like signals (planet candidates and eclipsing binaries) from stellar variability and instrumental noise with an average precision of $97.0 \%$ and an accuracy of $97.4 \%$. In vetting mode, the model is trained to identify only planet candidates with the help of newly added scientific domain knowledge, and achieves an average precision of $69.3 \%$ and an accuracy of $97.8 \%$.

In [14], the authors present a study of several classification models (SVM, KNN and RF) used to assign a probability of an observation being an exoplanet. A Random Forest Classifier was selected as the optimum machine learning model to classify objects of interest in the Cumulative Kepler Object of Interest (KOI) catalog using the table attributes as features. The Random Forest Classifier obtained a cross-validated accuracy score of $98.96 \%$, precision $99.55 \%$ and recall of $97.21 \%$ on the training set. 


\begin{tabular}{|c|c|c|c|c|}
\hline Ref & Catalog & Feature Extraction & ML Method & Performance \\
\hline [15] & $\begin{array}{c}\text { REAL } \\
\text { Kepler } \\
\text { Q1-Q17 DR24 }\end{array}$ & $\begin{array}{l}114 \text { Attributes } \\
\text { calculated }\end{array}$ & RF (3 classes) & $\begin{array}{c}\text { Accuracy: } \\
0,973\end{array}$ \\
\hline [16] & $\begin{array}{c}\text { REAL } \\
\text { Kepler } \\
\text { Q1-Q17 DR24 }\end{array}$ & $\begin{array}{l}\text { 1D folding curve: } \\
\text { global \& local view }\end{array}$ & $\begin{array}{c}\text { LLR } \\
\text { Fully connected NN } \\
\text { CNN }\end{array}$ & $\begin{array}{c}\text { Accuracy: } \\
0.917, \\
0.94 \\
0.958 \\
\text { AUC: } 0.963, \\
0.977 \\
0.988\end{array}$ \\
\hline [17] & $\begin{array}{c}\text { REAL } \\
\text { Kepler } \\
\text { Q1-Q17 DR24 }\end{array}$ & $\begin{array}{l}\text { 1D folding curve: } \\
\text { global \& local view } \\
\text { Centroid curves } \\
\text { Stellar } \\
\text { parameters }\end{array}$ & DCNN & $\begin{array}{l}\text { Accuracy: } \\
0.975 . \\
\text { Precision: } \\
0.955\end{array}$ \\
\hline [18] & $\begin{array}{c}\text { REAL } \\
\text { TESS } \\
1-5 \text { sector }\end{array}$ & $\begin{array}{l}\text { 1D folding curve: } \\
\text { global \& local view } \\
\text { Secondary } \\
\text { eclipse view }\end{array}$ & CNN for Triage & $\begin{array}{c}\text { Accuracy: } \\
0.974 . \\
\text { AUC: } \\
0.992 \\
\text { Precision: } \\
0.97\end{array}$ \\
\hline [14] & $\begin{array}{c}\text { REAL } \\
\text { Kepler } \\
\text { Cumulative }\end{array}$ & $\begin{array}{l}\text { Features from } \\
\text { interactive table }\end{array}$ & SVM, KNN, RF & $\begin{array}{c}\text { Accuracy: } \\
0.9896 . \\
\text { Precision: } \\
0.9955 \\
\text { Recall: } \\
0.9721 \\
\text { F1: } 0.9837\end{array}$ \\
\hline [19] & $\begin{array}{c}\text { REAL } \\
\text { Kepler } \\
\text { Q1-Q17 DR24 } \\
\text { TESS } \\
\text { 1-5 sector }\end{array}$ & $\begin{array}{l}\text { TSFresh } \\
789 \text { features }\end{array}$ & $\begin{array}{l}\text { Gradient } \\
\text { Boosted } \\
\text { trees }\end{array}$ & $\begin{array}{c}\text { Kepler AUC: } \\
0.948 . \\
\text { Recall: } \\
0.96 \\
\text { Precision: } \\
0.82 \\
\text { TESS AUC: } \\
0.80 . \\
\text { Recall: } \\
0.82 \\
\text { Precision: } \\
0.81\end{array}$ \\
\hline [20] & $\begin{array}{l}\text { SIMULATED } \\
\text { with tansit } \\
\text { REAL } \\
\text { without transit }\end{array}$ & $\begin{array}{l}50000 \text { lightcurves: } \\
25000 \text { with transit } \\
25000 \text { without transit }\end{array}$ & MLP, CNN & $\begin{array}{l}\text { Accuracy: } \\
0.99 . \\
\text { Recall: } \\
0.99\end{array}$ \\
\hline
\end{tabular}

Table 1: Machine learning approaches for exoplanet detection. 
In [19], the author present an approach based on a tree-based classifier using a popular machine learning tool 'lightgbm', to detect exoplanets using the transit method. They use time-series analysis library 'TSFresh' to extract 789 features from lightcurves. These features capture information about the characteristics of each lightcurve. This was tested on synthetic data which proved it to be more effective than conventional box least squares fitting (BLS). On Kepler data, the method is able to detect a planet transit with an AUC of $94.8 \%$ of accuracy and Recall of $96 \%$. With the TESS data, the method is able to classify lightcurves with an accuracy of $98 \%$ and is able to identify planets with a Recall of $82 \%$.

In [20] the authors present an approach based on CNN for detecting exoplanet transits. A 2D phase folding technique is proposed, generating a set of images for training. They test the method with five different types of deep learning models with or without folding. Synthetic lightcurves were generated as the input of these models. The results indicate that a combination of two-dimension convolutional neural network with folding is the best choice for the future transit analysis. All models with folding have accuracy above $98 \%$. The accuracy of models without folding can become about $85 \%$. The precision and recall have a similar trend. This article is based on this approach, the main difference is that it uses real data with transit for both training and testing.

\subsection{Real data description}

The dataset consists of Kepler observations of near 200,000 stars started from 2nd May 2009 to 11th May 2013. The data is divided in 18 quarters from Q0 to Q17. The length of each quarter is about 90 days, but some quarters are shorter. The data includes long and short cadence which took data every 30 and 2 minutes respectively. Long cadence data will be only considered from Q1-Q17 quarters because there are not enough stars observed in short cadence.

The Transit Planet Search (TPS) module carefully observes the light curves and identifies possible signals called Threshold Crossing Events (TCE). The Data Validation module creates reports based on the probability of veracity of the signals; then the Robovetter [21] examines the signals and creates a Kepler Objects of Interest (KOI) catalog. Those confirmed to have nothing to do with planetary transits are labeled as false positives (FP). The remain are called planet candidates (PC). NASA provides the list of all confirmed planet transits $(\mathrm{CP})$ as well the planet and star properties.

The Cumulative Kepler Objects of Interest (KOI) table provides the most accurate dispositions and stellar and planetary information for all KOIs in one place. The KOI catalog table contains unique object of interest identifiers, exoplanet archive information, transit properties, among others threshold-crossing events properties. The labels were sourced from the catalog's koi_disposition column as the ground truth. The catalog contains 9564 KOIs, out of which 2358 are confirmed exoplanets, 2366 remain candidates and the rest (4840 objects) are false positives. The last group of objects was removed from the dataset. Since since it is searching for signals with planet transits, the candidates and confirmed exoplanets have both been combined into the transit labelled data presented in the next sections.

The non-transit labelled data were obtained from the Kepler Data Release 25-Q1 (DR25) table and consists of $43273 \mathrm{KOIs}$ with no transit.

Two observed fluxes columns are used from each observation data: one is the simple aperture photometry (SAP) which is the flux obtained by direct photometry analysis and can include some other device; the other one is the Pre-search Data Conditioning (PDC) which represents a processed version of SAP where the devices are removed almost completely [22]. PDC light curves will be used. Since the unusual values produced by astrophysics events such as solar flares and micro lenses are not eliminated of the PDC light curve, all the points over 6 times standard deviation will be removed.

Since many of confirmed planets share star with other planets, the systems with only one confirmed planet or PC was chosen. Following the approach from [20], the main idea is to use a light curve with enough samples to represent 10 periods, considering that at 


\begin{tabular}{cc}
\hline Parameter & Value \\
\hline$\tau$ & 0.85 to 8.5 [days] \\
$a / r_{s}$ & 2 to 35 \\
$r_{p} / r_{s}$ & 0.005 to 0.4 \\
$i$ & 85 to 90 [deg] \\
$u_{1}$ & 0.210 to 0.731 \\
$u_{2}$ & 0.035 to 0.442 \\
\hline
\end{tabular}

Table 2: Transit parameters

bigger transit period, the higher amount of samples. At Kepler observations case, a curve has about 4320 samples taken each 30 minutes then the maximum allowed period to cover the 10 segments is 9 days. Therefore all transits with a period between 0.85 and 8.5 days were considered. On this point there are 583 light curves of the Q1 quarter, in order to maintain balance, the same amount of non-transit light curves was selected.

\subsection{Synthetic Data generation}

The method proposes to include a set of transit synthetic light curves in order to improve the performance of the classifier. Those are generated using the quadratic model for the limb darkening laws introduced analytically by Mandel \& Agol on [23]. The flux $f$, for a transit over a stellar disk with quadratic limb darkening is:

$$
f(k, z)=1-\frac{(1-c) \lambda_{e}(k, z)+c \lambda_{d}(k, z)+u_{2} \eta_{d}(k, z)}{1-u_{1} / 3-u_{2} / 6}
$$

where $k$ is the radius ratio, $z$ is the projected distance, $c=u_{1}+2 u_{2}, u_{1}$ and $u_{2}$ are the quadratic limb darkening coefficients, and $\lambda_{e}, \lambda_{d}$ and $\eta_{e}$ are functions of $k$ and $z$ defined in [23]. This model is implemented on the PyTransit Python library [24] with related parameters like the transit period $\tau$, the ratio of planet radius to stellar radius $\left(r_{p} / r_{s}\right)$, the ratio of orbital semimajor axis to stellar radius $\left(a / r_{s}\right)$ and the orbit inclination $(i)$. The values of those parameters were set the same as [20], table 2 gives a summary of them.

\subsection{Feature extraction}

\subsubsection{Lightcurves pre-processing}

The main purpose of this method is to find transits, so the light curves are preprocessed to first remove the empty intervals. The missing values are replaced with the average of the neighborhood of the empty interval. Noise with less than $10 \%$ of magnitude is added on this new values for a better consistency with reality. The light curves were interpolated to 4000 points i.e. 10 periods of 400 points each. Finally the data is normalized to have values between 0 and 1 .

\subsubsection{D Phase folding}

Related work on light curve feature extraction includes the phase folding technique introduced by [11] to take advantage of transit periodicity. It consists on folding each light curve on the transit period from the catalog and binning it to generate a $1 \mathrm{D}$ vector of a enhanced signal. This method increase the transit detection but the transit period has to be known in advance, otherwise the folding period can differ from it and the transit will be undetectable for the model. The above condition represents a difficulty when it is wanted to search for transits on new released observational data. To solve this problem [20] presents a phase folding method that generates a $2 \mathrm{D}$ representation by folding each light curve on a period that can be different from the transit period, improving the transit detection regardless of the transit and folding period. Following this method, the detection model inputs were generated by folding the light curve in 10 segments using the transit period from the catalog's koi_period column, then the values for each period were incorporated as rows of an image. 
Fig. 1 shows the 2D phase folding proccess on three examples of lightcurves: column (a) shows a synthetic lightcurve with transit folded on the transit period, column (b) shows a real lightcurve with unsuitable folding for the actual transit period, and column (c) presents a real lightcurve without transit. First row of the figure presents the pre-processed light curve, a 1D signal of 4000 samples normalized to have values between 0 and 1 . Ten folds of 400 samples each are enumerated. Second row of the figure shows a zoom view of each fold, the transit is visible on (a) at the same phase on each fold, since (b) has a folding period different from the transit period, the drops on each fold appears to be shifted to a lightly different phase than in the previous one, on (c) no transit drop is visible on any fold. Third row of the figure presents the mean of the 10 folds on each case, this would be the inputs of the [11] proposed model, i.e. a 1D vector of 400 samples. The transit is visible on (a) closer to the 350 sample, on (b) the mean transit signal becomes unclear and the model may not give a correct answer on transit detection, confusing it with the one presented in (c). Finally, fourth row shows the 2D representation of the folded lightcurve, a $10 \times 400$ pixels image which is the input of the detection model. The dark bands indicating the transit are visible on (a) and (b). Therefore, a model may be able to detect transits successfully and distinguish it from the one in (c).
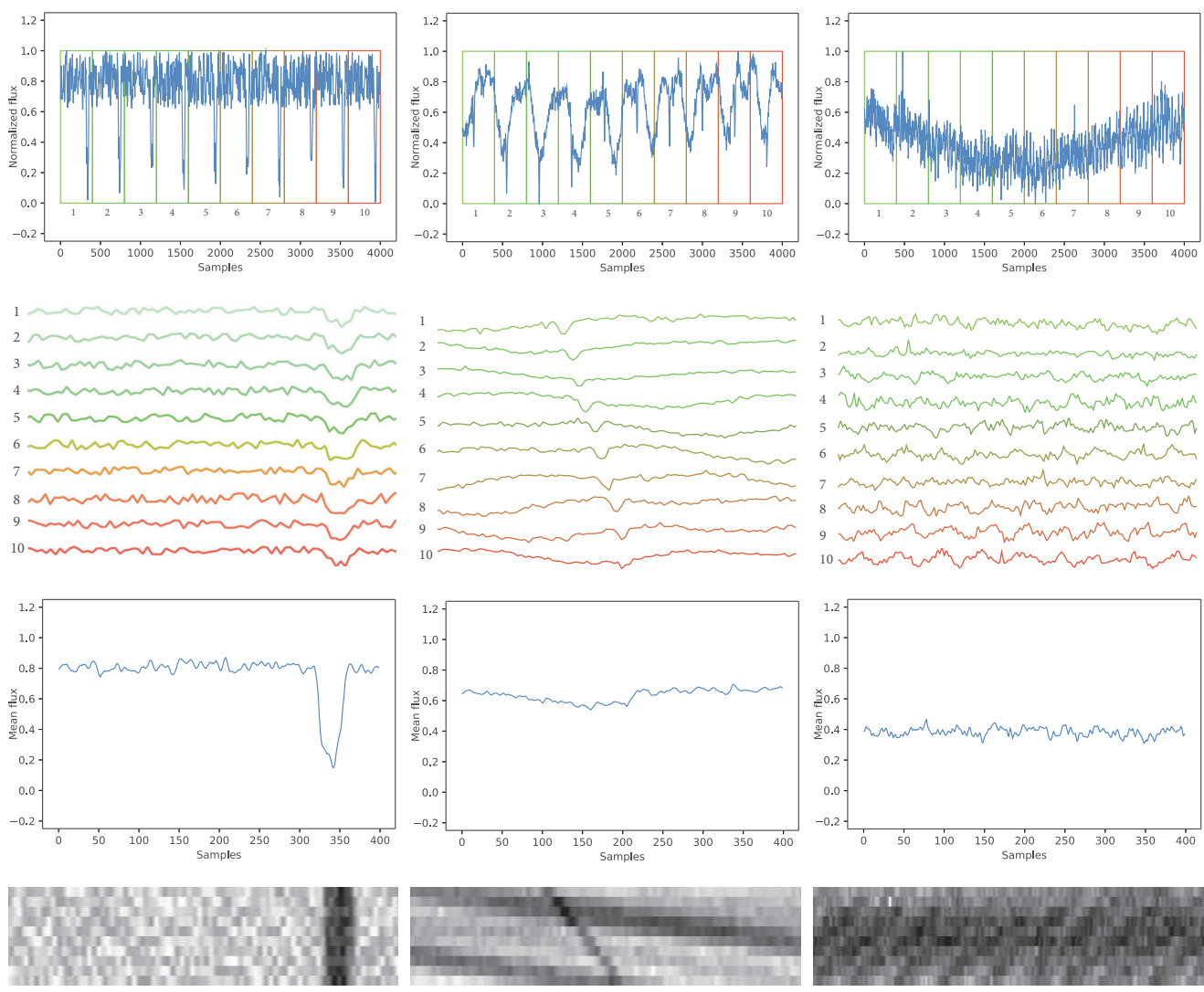

Figure 1. 2D Phase folding for three cases: Synthetic lightcurve (left). Real lightcurve with transit (center). Real lightcurve without transit (right).

\subsection{Detection model}

The classification task consists on sort out every light curve into two categories: Planet candidate and False positive. Different deep learning approaches have been used for this application, however convolutional neural network was chosen since they outperform artificial neural networks in classification tasks where the data is spatially aligned such as image or audio [25]. It is because CNN leverages the spatial structure of the output detecting local features which only need to be learned once, therefore the number of 
trainable parameters, the memory usage, and the number of computations of the desired output will decrease.

Transfer learning takes a large network that has already been trained for a specific problem and then fits it to a new problem. This adjust is performed at the end of the network, modifying the number of output neurons to match the number of classes of the new problem (2 classes). This is a very useful technique since the first stages of the network usually recognize general features that can be applied to almost every classification problem [26]. Clearly it is necessary to perform train in order to adapt the last layer to the new classes, but thanks to transfer learning it is not necessary to train the whole network again. In fact one can choose which layers to train and which not to train. This is very efficient when considering the computational cost of training a network of this magnitude.

The proposed neural network architecture is based on Xception developed by Francois Chollet [27]. The pre-trained network with 71 deep layers has learned rich feature representations for a wide range of images and. It can classify 1000 categories of objects and has an image input size of 299x299. It is necessary to modify the input dimension of the network and the number of output neurons. In this binary classification problem a monocromatic image input size of 400x10 and one neuron on the output layer with a sigmoid function of activation were implemented. The output $y$ of the model depends on the neural network decision threshold $T$. This threshold determines the minimum classification probability on which the light curve will be classified as a planet candidate (the probability predicted is greater than $T$ ) or as an false positive (the probability predicted is smaller than $T$ ) as shown Eq. 2 where $z$ is the weighted sum on the inputs.

$$
y= \begin{cases}1 & \text { if } \frac{1}{1+e^{-z}} \geq T \\ 0 & \text { otherwise }\end{cases}
$$

\subsection{Training the model}

In order to see the effect of increasing synthetic data on training, DCNN models were built with $R=483$ real curves and a ratio of synthetic curves $S$ with transit defined by the $\lambda$ parameter Eq. 3 shows. Additionally, the same number $S+R$ of non transit curves are added to the training set to maintain balance. Fig. 2.a summarizes the workflow from the training stage.

$$
S=\frac{\lambda R}{100-\lambda}
$$

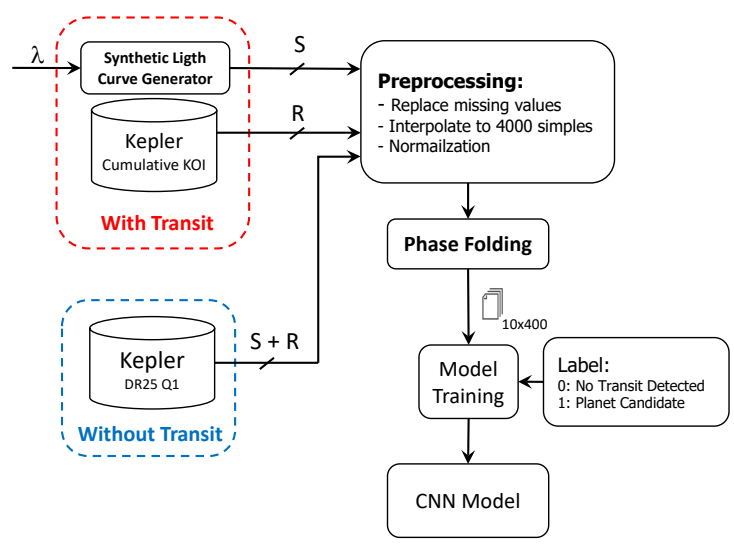

a) Training stage

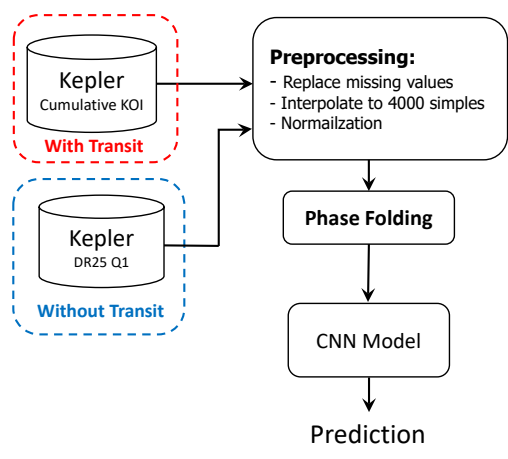

b) Validation stage

Figure 2. Training and validation stages of the proposed method. 


\begin{tabular}{ccclccccc}
\hline & \multicolumn{3}{l}{ With transit } & \multicolumn{3}{l}{ Without transit } & \multicolumn{3}{c}{ Metrics } \\
\hline Scenario & $S$ & $R$ & $S$ & $R$ & $T$ & $F_{1}$ & TPR & Precision \\
\hline 1 & 0 & 483 & 0 & 483 & 0.5 & 0.743 & 0.740 & 0.747 \\
2 & 483 & 0 & 0 & 483 & 0.5 & 0.206 & 0.120 & 0.750 \\
\hline
\end{tabular}

Table 3: Detection performance under proposed scenarios

\subsection{Evaluation Metrics}

For evaluation purposes, $R=100$ real transit light curves and $R=100$ non-transit light curves was selected as test set. This work uses the following metrics to asses the performance of the CNN model based on the workflow shown in Fig. 2 (b):

- Accuracy: The portion of correct classifications.

$$
\text { accuracy }=\frac{T P+T N}{T P+F P+T N+F N}
$$

- Precision: The ratio of lightcurves classified as planet candidates that are true planet candidates, also known as reliability.

$$
\text { precision }=\frac{T P}{T P+F P}
$$

- $\quad$ True Positive Rate (TPR): The ratio of true planet candidates that are classified as planet candidates, also known as recall.

$$
T P R=\frac{T P}{T P+F N}
$$

- $\quad$ False Positive Rate (FPR): The ratio of non transit lightcurves misclassified as planet candidates.

$$
F P R=\frac{F P}{F P+T N}
$$

- Finally, $F_{1}$-Score is calculated as shown in Eq. 8 and is used to evaluate in a single value the combination of both precision and recall.

$$
F_{1}=2 \times \frac{\text { precision } \times \text { recall }}{\text { precision }+ \text { recall }}
$$

\section{Experimental results}

The values of the accuracy, precision and TPR depend on the the neural network threshold $T$ chosen for the model see Eq. 2 . The main hypothesis of this work is that the performance of the model can be also improved using a ratio $\lambda$ of synthetic lightcurves on the training stage.This section presents the ratio selection of synthetic lightcurves using a coarse and a fine tunning, also a comparison between the proposed method and the ones reported in the literature is presented.

\subsection{Best $\lambda$ ratio selection}

The detection performance was tested on a test set of $R=100$ real curves with transit and the same amount of real curves without transit, on two extreme scenarios:

1. Training with only real lightcurves with transit $(S=0, R=483)$ and $S+R$ real lightcurves without transit.

2. Training with only synthetic lightcurves with transit $(S=483, R=0)$ and $S+R$ real lightcurves without transit. 


\begin{tabular}{ccc}
\hline Parameter & Description & Value \\
\hline Encode $S$ & Two decimals & 11 bits \\
Encode $U$ & Encoded $S$ and $U$ concatenated & 7 bits \\
Chromosome size & Number of chromosomes in one generation & 10 \\
Population size & Iterations & 50 \\
Number of generations & Tournament between parents & 3 \\
Selection & Single point at the middle & 0.9 \\
Crossover type and rate & Random bit flip & 0.1 \\
Mutation type and rate & Determines members that survives & $F_{1}$ \\
Fitness function & &
\end{tabular}

Table 4: GA implementation details

On both scenarios the neural network threshold is fixed on $T=0.5$ due to the fact that this is a binary classification problem where 0 is a predicted lightcurve without transit and 1 is a predicted planet candidate.

Table 3 shows the performance of the models built for each scenario. It is observed that the model trained with only real curves can detect the $74 \%$ of the transit light curves from the test set with a precision of $74.7 \%$; this is a good rate however underperform the reported metrics from the literature. On the other hand when the model is trained with synthetic curves it can detect the transit lightcurves with a similar level of precision than the model trained with real curves, but clearly it does not have all the variability of curves with transit.

Therefore, it can be concluded that training with real curves provides variability to the model on transit lightcurves detection but incorporate synthetic light curves can improve the precision of the prediction. In order to find the best ratio of $\lambda$ and $T$, a coarse tunning with an heuristic optimization method and a sensibility analysis are proposed.

\subsection{Heuristic search of optimal parameters}

The optimization problem is described on Eq. 9, the amount of synthetic lightcurves $S$ and the neural network threshold $T$ are the decision variables.

$$
\begin{array}{cl}
\underset{S, T}{\operatorname{maximize}} & \mathrm{F}_{1}(\lambda, T) \\
\text { subject to } & 0 \leq \lambda \leq 80 \% \\
& 0<T<1
\end{array}
$$

The fitness function is the balanced $F_{1}$-score, which is the harmonic mean between precision and TPR calculated on Eq. 5 and 6 respectively where:

- $\quad$ True Positive (TP): lightcurve with transit detected as planet candidate.

- $\quad$ False Positive (FP): lightcurve without transit detected as planet candidate.

- $\quad$ True Negative (TN): lightcurve without transit detected as false positive.

- $\quad$ False Negative (FN): lightcurve with transit detected as false positive.

Genetic algorithms have been widely used in the last decades, because they are considered a tool to solve complex optimization problems managing the influence of the uncertainties of typical design engineering scenarios. The main idea behind GA is to evolve a population of chromosomes (possible candidate solutions of the problem), in several iterations (also called generations), using operators such as crossover and mutation and evaluated under a fitness function. In this context, this article uses GA as an optimization tool to determine the optimal ratio of artificial curves and the optimal value of the neural network threshold. Table 4 summarizes the parameters settings used for the GA implementation.

Table 5 shows the results of GA applied on different settings. The first column contains the population size. The second column shows the number of generations/iterations of 
the algorithm. The third contains the amount of synthetic curves with transit used on training stage. The fourth column shows the neural network decision threshold. The fifth column contains the values of $F_{1}$ on each experiment and finally the sixth column shows the number of $F_{1}$ calculations i.e the amount of models trained in order to get that $F_{1}$ score value. The best value of $F_{1}(0.9801)$ is obtained for three different configurations: 1) 10 chromosomes and 50 generations, 2) 20 chromosomes and 20 generations and 3) 50 chromosomes and 10 generations. The three of them have the same value of $S$ (1403), this value corresponds to $\lambda=74.3 \%$ of synthetic lightcurves and $27.7 \%$ real curves with transit on training stage. The value of $T$ varies between 0.21 and 0.23 so there is no big difference between the three of them. The configuration 1) was chosen because it is the one that trains the fewest models to obtain the same result and it also has the highest threshold $T$.

\begin{tabular}{cccccc}
\hline Population & \#Generations & $\mathbf{S}$ & $\mathbf{T}$ & $\mathbf{F}_{1}$ & \#F $_{1}$ calc. \\
\hline \multirow{4}{*}{$\mathbf{1 0}$} & 5 & 1633 & 0.09 & 0.9607 & 80 \\
& 10 & 1173 & 0.07 & 0.9371 & 160 \\
& 15 & 1334 & 0.41 & 0.9591 & 240 \\
& 20 & 759 & 0.28 & 0.9607 & 320 \\
$\mathbf{4} \mathbf{2 0}$ & $\mathbf{5 0}$ & $\mathbf{1 4 0 3}$ & $\mathbf{0 . 2 3}$ & $\mathbf{0 . 9 8 0 1}$ & $\mathbf{8 0 0}$ \\
& 5 & 1794 & 0.10 & 0.9560 & 180 \\
& 10 & 1403 & 0.28 & 0.9751 & 380 \\
& 15 & 1403 & 0.19 & 0.9753 & 540 \\
& $\mathbf{2 0}$ & $\mathbf{1 4 0 3}$ & $\mathbf{0 . 2 2}$ & $\mathbf{0 . 9 8 0 1}$ & $\mathbf{8 2 0}$ \\
$\mathbf{5 0}$ & 50 & 1794 & 0.1 & 9.5609 & 1800 \\
& 5 & 1334 & 0.23 & 0.9651 & 440 \\
& $\mathbf{1 0}$ & $\mathbf{1 4 0 3}$ & $\mathbf{0 . 2 1}$ & $\mathbf{0 . 9 8 0 1}$ & $\mathbf{8 8 0}$ \\
& 15 & 1403 & 0.19 & 0.9753 & 1320 \\
& 20 & 1403 & 0.18 & 0.9705 & 1760 \\
& 50 & 1403 & 0.2 & 0.9753 & 4400 \\
\hline
\end{tabular}

Table 5: GA results for different parameter settings.

\subsection{Sensitivity analysis}

A fine tunning is performed by analyzing the dependence of the $F_{1}$ score value on the values of $\lambda$ and $T$. The $\lambda$ value is ranged between 0 and $80 \%$, increasing $S$ from 0 to 1932 in steps of 23. This provides more resolution between $60 \leq \lambda \leq 80 \%$ since the best ratio according to the previous section is between this range. The threshold value $T$ is ranged from 0 to 1 using two decimals. Each model is trained using $R=483$ real lightcurves with transit, $S$ synthetic lightcurves and $S+R$ real lightcurves without transit to mantain balance. The value of $F_{1}$ is calculated for each model with 200 real lightcurves, half of them with transit and the other half without transit. Fig. 3 presents a 3D plot of the $F_{1}$ score for each pair of $\lambda$ and $T$ values. It can be observed that for $\lambda$ greater than $50 \%$ the value of $F_{1}$ is higher, which proves the hypothesis that increasing the number of synthetic lightcurves improves detection performance. It can also be observed that for $\lambda$ between $72 \%$ and $77 \%$ and for $T$ between 0.1 and 0.4 the highest $F_{1}$ values are obtained, with the maximum visible value in $\lambda \approx 74 \%$ and $T \approx 0.2$, which is consistent with the optimum value found in the previous section.

To get a broader view of the effect of the decision threshold $T$, models were trained in the same way as the above by ranging the value of $\lambda$ between 0 and $80 \%$ in steps of $5 \%$. For each ratio, the threshold $T$ was varied and TPR and FPR were calculated to construct the Receiver Operating Characteristic Curve (ROC) in Fig. 4.

It shows that increasing the threshold $T$ increases the rate of true positives, but tends to misclassify negative instances, so the threshold value for which the roc curve is closest to the ideal case (FPR $=0, T P R=1)$ must be found, this value will be denoted as the best threshold . It can be seen that the curve of ratio $\lambda=70 \%$ has the point $(0.050,0.970)$ that 


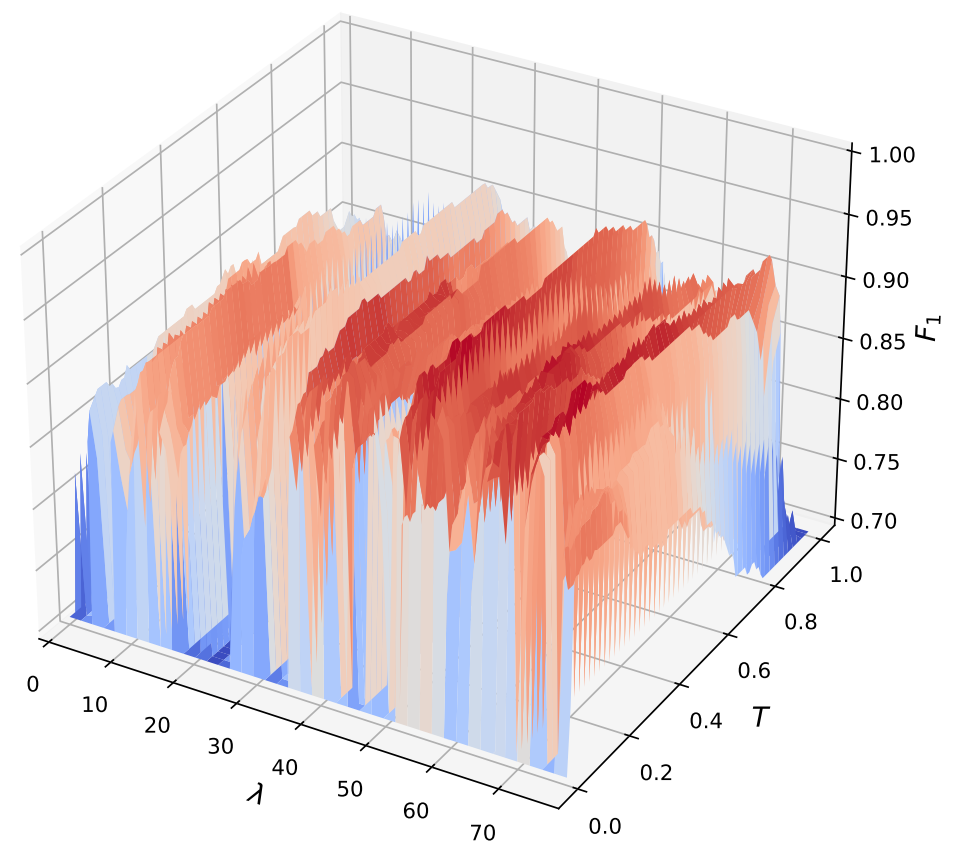

Figure 3. 3D plot of $F_{1}$ against ratio $\lambda$ and threshold $T$

is the closest to the ideal one, which is consistent with the value presented in the coarse adjustment with GA.

Table 6 shows the results obtained for each ratio. First column presents the ratio $\lambda$ in percentage. The second column shows the respective number of synthetic light curves $S$. Third column presents the value of the threshold $T$ closer to the ideal ROC curve point. Columns 4 to 9 show the evaluation metrics described on section 2.6.

It can be observed that from first limit scenario where $\lambda=0$ (see table 3 ), increasing only the threshold $T$ from 0.5 to 0.608 , the precision is improved from 0.747 to 0.923 and thus the $F_{1}$ value from 0.743 to 0.808 .

The TPR value, i.e. those light curves with transit that are correctly detected, starts to increase from $\lambda=25 \%$. This means that adding synthetic curves add knowledge to the model and helps it to more easily identify real curves with transit. On the other hand, the precision starts to increase from $\lambda=60 \%$. This implies that adding synthetic curves can improve the detection precision, however this generates a bias in the model since it is difficult for it to detect the real light curves with transit, so it is necessary to decrease the decision threshold $T$ that separates the two classes.

\subsection{Comparison with related work}

The best model was obtained with $\lambda=74.3 \%$ and $T=0.23$ (See section 3.2). To evaluate the model $R=200$ real lightcurves (100 with transit, 100 without transit) were used, achieving a precision of 0.9705 , a TPR of 0.99 , a $F_{1}$ of 0.9801 , a FPR of 0.03 and an accuracy of 0.98 . Given the wide range of databases, the comparison between the presented approach and the related work will be centered on two works from the table 1: The one presented in [14] in order to compare two different approaches on the same dataset and the 


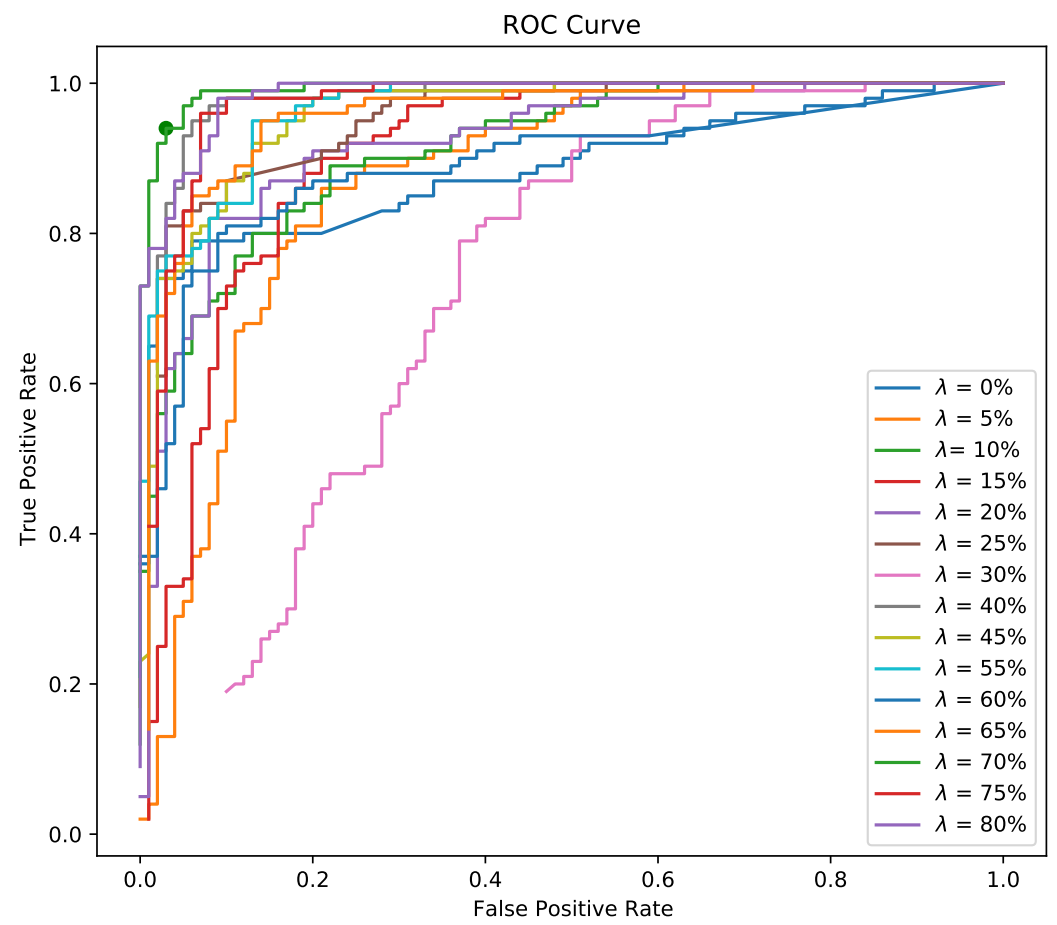

Figure 4. ROC curve of every ratio

one presented in [20], in order to compare the effect of the same approach on both real and synthetic data.

Table 7 presents the metrics comparison between the proposed approach and the related work described. Column 2 to 5 show the metrics obtained during training ans the rest of them show the metrics obtained during test stage. In the case of [14] which uses real data to train the model, the metrics available correspond only to the training stage; this approach does not allow to properly analyse the performance of the model with new data. The expected performance of this model evaluated on unknown real lightcurves should be the one from first scenario presented on Table 3.

On the other hand, the article [20] presented validation metrics but the model is trained and evaluated on synthetic light curves only. In order to perform a fair comparison between our approach and this work, the model in [20] has been reproduced and evaluated with real lightcurves, obtaining a precision of 0.5 , a TPR of 0.01 , a $F_{1}$ of 0.0196 , a FPR of 0.01 , and an accuracy of 0.5 . This performance is very similar than the second scenario presented on Table 3 where the model is unable to detect a real lightcurve with transit since the real transits may not have such a relevant drop in the flux. However the higher value of the precision shows that adding synthetic lightcurves to the training stage brings knowledge to the model. In this case the method proposed outperforms the ones reported on the literature and demonstrate that combining real and synthetic lightcurves on the training stage can improve the detection metrics.

\section{Conclusion}

In this paper, the development of a deep learning system for detecting planetary transits in Kepler Telescope lightcurves is presented. The approach is based on related work from the literature and enhanced to validation with real lightcurves. 2D phase folding is used as a feature extraction method that allows real and synthetic lightcurves with transit to be described by an image distinguishable from those without transit. The 


\begin{tabular}{ccccccccc}
\hline$\lambda(\%)$ & $\mathbf{S}$ & $\mathbf{T}$ & Accuracy & Precision & TPR & FPR & $\mathbf{F}_{\mathbf{1}}$ & FNR \\
\hline 0 & 0 & 0.608 & 0.860 & 0.923 & 0.720 & 0.060 & 0.808 & 0.280 \\
5 & 25 & 0.639 & 0.825 & 0.825 & 0.850 & 0.210 & 0.837 & 0.150 \\
10 & 60 & 0.953 & 0.835 & 0.818 & 0.900 & 0.130 & 0.857 & 0.100 \\
15 & 85 & 0.394 & 0.840 & 0.876 & 0.920 & 0.210 & 0.897 & 0.080 \\
20 & 121 & 0.631 & 0.870 & 0.922 & 0.830 & 0.080 & 0.873 & 0.170 \\
25 & 161 & 0.999 & 0.885 & 0.824 & 0.940 & $\mathbf{0 . 0 3 0}$ & 0.878 & 0.060 \\
30 & 207 & 0.608 & 0.710 & 0.810 & 0.940 & 0.370 & 0.870 & 0.060 \\
35 & 260 & 0.456 & 0.850 & 0.873 & $\mathbf{0 . 9 7 0}$ & 0.070 & 0.919 & $\mathbf{0 . 0 3 0}$ \\
40 & 322 & 0.963 & 0.945 & 0.873 & $\mathbf{0 . 9 7 0}$ & 0.080 & 0.919 & $\mathbf{0 . 0 3 0}$ \\
45 & 395 & 0.685 & 0.895 & 0.882 & 0.900 & 0.130 & 0.891 & 0.100 \\
50 & 483 & 0.578 & 0.840 & 0.897 & 0.880 & 0.040 & 0.888 & 0.120 \\
55 & 590 & 0.727 & 0.910 & 0.782 & 0.720 & 0.130 & 0.750 & 0.280 \\
60 & 730 & 0.765 & 0.855 & 0.932 & $\mathbf{0 . 9 7 0}$ & 0.090 & 0.950 & $\mathbf{0 . 0 3 0}$ \\
65 & 897 & 0.444 & 0.905 & 0.938 & 0.910 & 0.140 & 0.923 & 0.090 \\
70 & 1127 & 0.234 & $\mathbf{0 . 9 6 0}$ & $\mathbf{0 . 9 5 0}$ & $\mathbf{0 . 9 7 0}$ & 0.050 & $\mathbf{0 . 9 6 0}$ & $\mathbf{0 . 0 3 0}$ \\
75 & 1450 & 0.561 & 0.940 & 0.940 & 0.950 & 0.060 & 0.945 & 0.050 \\
80 & 1932 & 0.141 & 0.945 & 0.872 & 0.960 & 0.090 & 0.914 & 0.040 \\
\hline
\end{tabular}

Table 6: Sensivity analysis results

\begin{tabular}{ccccccccc}
\hline \multicolumn{3}{c}{ Training } & \multicolumn{5}{c}{ Test } \\
\hline Ref & Accuracy & Precision & TPR & $F_{1}$ & Accuracy & Precision & Recall & $F_{1}$ \\
\hline$[14]$ & 0.989 & 0.995 & 0.972 & 0.983 & & & & \\
{$[20]$} & 0.500 & 0.500 & 0.010 & 0.019 & 0.500 & 0.500 & 0.010 & 0.019 \\
Our & 0.980 & 0.970 & 0.990 & 0.980 & 0.980 & 0.970 & 0.990 & 0.980 \\
\hline
\end{tabular}

Table 7: Comparison of the proposed approach with related work

model parameters are adjusted to improve the performance of the classification. The method is evaluated on real lightcurves from the Kepler's catalog and demonstrates superior performance against other approaches presented on the state of art.

The main contribution of this work is the enhance of a detection model including the generation of synthetic lightcurves with transit from estimated parameters. The best ratio of synthetic data is founded using a coarse tunning with Genetic algorithms and evidenced with a sensibility analysis. The evaluated metrics demonstrate that the combination of real and synthetic lightcurves with transit on the training stage add knowledge to the model and improve the performance on real light curves.

Future work will consider extend the study to systems with more than one confirmed planet or planetary candidate dealing with multi-transit detection on the same lightcurve. Also the implementation of the method on a different database like the NASA's Transitig Exoplanet Survey Satellite (TESS), mission that has discover already 166 exoplanets and has 4604 planet candidates.

1 Author Contributions: Conceptualization, S.C., P.G., M.C., and G.F.; methodology, S.C., M.C., and 2 G.F.; software, S.C.; validation, S.C., P.G., and G.F.; formal analysis, M.C., and G.F.; investigation,

H.V.; resources, G.F.; data curation, S.C., and P.G.; writing-original draft preparation, S.C., and

E.F.; writing-review and editing, E.F., S.D., and H.V.; visualization, S.C.; supervision, G.F.; project administration, S.D.; funding acquisition, G.F. All authors have read and agreed to the published

version of the manuscript.

7 Funding: This research was supported in part by the Chilean Ministry of Education under Project FONDECYT 1191188.

9 Conflicts of Interest: The authors declare no conflict of interest. 


\section{References}

1. NASA. Exoplanet Exploration Program, 2021.

2. Weidemann, V. The Atmosphere of the White Dwarf Van Maanen 2. The Astrophysical Journal 1960, 131, 638.

3. Mayor, M.; Queloz, D. A Jupiter-mass companion to a solar-type star. Nature 1995, 378, 355-359.

4. Médjahdi, K. Radial velocity of a sound source in circular motion for illustrating the detection of an exoplanet. American Journal of Physics 2020, 88, 814-818.

5. Wright, J.T.; Gaudi, B.S. Exoplanet detection methods. arXiv preprint arXiv:1210.2471 2012.

6. Borucki, W.J. KEPLER Mission: development and overview. Reports on Progress in Physics 2016, 79, 036901.

7. Ricker, G.R.; Latham, D.; Vanderspek, R.; Ennico, K.; Bakos, G.; Brown, T.; Burgasser, A.; Charbonneau, D.; Clampin, M.; Deming, L.; others. Transiting exoplanet survey satellite (TESS). American Astronomical Society Meeting Abstracts \# 215, 2010, Vol. 215, pp. 450-06.

8. Thompson, S.E.; Mullally, F.; Coughlin, J.; Christiansen, J.L.; Henze, C.E.; Haas, M.R.; Burke, C.J. A machine learning technique to identify transit shaped signals. The Astrophysical Journal 2015, 812, 46.

9. McCauliff, S.D.; Jenkins, J.M.; Catanzarite, J.; Burke, C.J.; Coughlin, J.L.; Twicken, J.D.; Tenenbaum, P.; Seader, S.; Li, J.; Cote, M. Automatic Classification of Kepler Planetary Transit Candidates. The Astrophysical Journal 2015, 806, 6. doi:10.1088/0004$637 \mathrm{x} / 806 / 1 / 6$.

10. Schanche, N.; Cameron, A.C.; Hébrard, G.; Nielsen, L.; Triaud, A.H.M.J.; Almenara, J.M.; Alsubai, K.A.; Anderson, D.R.; Armstrong, D.J.; Barros, S.C.C.; Bouchy, F.; Boumis, P.; Brown, D.J.A.; Faedi, F.; Hay, K.; Hebb, L.; Kiefer, F.; Mancini, L.; Maxted, P.F.L.; Palle, E.; Pollacco, D.L.; Queloz, D.; Smalley, B.; Udry, S.; West, R.; Wheatley, P.J. Machine-learning approaches to exoplanet transit detection and candidate validation in wide-field ground-based surveys. Monthly Notices of the Royal Astronomical Society 2018, 483, 5534-5547, [https:/ /academic.oup.com/mnras/article-pdf/483/4/5534/27496900/sty3146_supplemental_file.pdf]. doi:10.1093/mnras/sty3146.

11. Pearson, K.A.; Palafox, L.; Griffith, C.A. Searching for exoplanets using artificial intelligence. Monthly Notices of the Royal Astronomical Society 2018, 474, 478-491.

12. Armstrong, D.J.; Pollacco, D.; Santerne, A. Transit shapes and self-organizing maps as a tool for ranking planetary candidates: application to Kepler and K2. Monthly Notices of the Royal Astronomical Society 2016, 465, 2634-2642, [https:/ / academic.oup.com/mnras/article-pdf/465/3/2634/8420208/stw2881.pdf]. doi:10.1093/mnras/stw2881.

13. Priyadarshini, I.; Puri, V. A convolutional neural network (CNN) based ensemble model for exoplanet detection. Earth Science Informatics 2021, pp. 1-13.

14. Sturrock, G.C.; Manry, B.; Rafiqi, S. Machine Learning Pipeline for Exoplanet Classification. SMU Data Science Review 2019, 2, 9.

15. Catanzarite, J.H. Autovetter Planet Candidate Catalog for Q1-Q17 Data Release 24, (KSCI-19090-001). Technical report, NASA Ames Research Center, 2015.

16. Shallue, C.J.; Vanderburg, A. Identifying Exoplanets with Deep Learning: A Five-planet Resonant Chain around Kepler-80 and an Eighth Planet around Kepler-90. The Astronomical Journal 2018, 155, 94. doi:10.3847/1538-3881/aa9e09.

17. Ansdell, M.; Ioannou, Y.; Osborn, H.P.; Sasdelli, M.; Smith, J.C.; Caldwell, D.; Jenkins, J.M.; Räissi, C.; Angerhausen, D.; and. Scientific Domain Knowledge Improves Exoplanet Transit Classification with Deep Learning. The Astrophysical Journal 2018, 869, L7. doi:10.3847/2041-8213/aaf23b.

18. Yu, L.; Vanderburg, A.; Huang, C.; Shallue, C.J.; Crossfield, I.J.M.; Gaudi, B.S.; Daylan, T.; Dattilo, A.; Armstrong, D.J.; Ricker, G.R.; Vanderspek, R.K.; Latham, D.W.; Seager, S.; Dittmann, J.; Doty, J.P.; Glidden, A.; Quinn, S.N. Identifying Exoplanets with Deep Learning. III. Automated Triage and Vetting of TESS Candidates. The Astronomical Journal 2019, 158, 25. doi:10.3847/1538$3881 / \mathrm{ab} 21 \mathrm{~d} 6$.

19. Malik, A.; Moster, B.P.; Obermeier, C. Exoplanet Detection using Machine Learning, 2021, [arXiv:astro-ph.EP/2011.14135].

20. Chintarungruangchai, P.; Jiang, I.G. Detecting Exoplanet Transits through Machine-learning Techniques with Convolutional Neural Networks. Publications of the Astronomical Society of the Pacific 2019, 131, 064502. doi:10.1088/1538-3873/ab13d3.

21. Thompson, S.E.; Coughlin, J.L.; Hoffman, K.; Mullally, F.; Christiansen, J.L.; Burke, C.J.; Bryson, S.; Batalha, N.; Haas, M.R.; Catanzarite, J.; others. Planetary candidates observed by Kepler. VIII. A fully automated catalog with measured completeness and reliability based on data release 25. The Astrophysical Journal Supplement Series 2018, 235, 38.

22. Smith, J.C.; Stumpe, M.C.; Van Cleve, J.E.; Jenkins, J.M.; Barclay, T.S.; Fanelli, M.N.; Girouard, F.R.; Kolodziejczak, J.J.; McCauliff, S.D.; Morris, R.L.; others. Kepler presearch data conditioning II-A bayesian approach to systematic error correction. Publications of the Astronomical Society of the Pacific 2012, 124, 1000.

23. Mandel, K.; Agol, E. Analytic light curves for planetary transit searches. The Astrophysical Journal Letters 2002, 580 , L171.

24. Parviainen, H. PYTRANSIT: fast and easy exoplanet transit modelling in PYTHON. Monthly Notices of the Royal Astronomical Society 2015, 450, 3233-3238.

25. Ciresan, D.C.; Meier, U.; Masci, J.; Gambardella, L.M.; Schmidhuber, J. Flexible, high performance convolutional neural networks for image classification. Twenty-second international joint conference on artificial intelligence, 2011.

26. Torrey, L.; Shavlik, J. Transfer learning. In Handbook of research on machine learning applications and trends: algorithms, methods, and techniques; IGI global, 2010; pp. 242-264.

27. Chollet, F. Xception: Deep learning with depthwise separable convolutions. Proceedings of the IEEE conference on computer vision and pattern recognition, 2017, pp. 1251-1258. 